\title{
Unmixing the cell
}

t's an interesting sociological question why the topic we might call the materials science of the living cell has taken so long to cohere. The answer perhaps lies in a tendency to associate organization with information, plans and blueprints. That the cell is an organized structure has been recognized at least since the start of the twentieth century. But from the mid-century, with the recognition that DNA encodes information, the dominant paradigm was one in which all aspects of life were orchestrated by genes.

Scientists interested in cell membrane biophysics, however, have long known that those lipid structures have an organizational repertoire of their own that needs no genetic guidance, and that this includes disordered and ordered phases both of which are found in cells. That is simply one example of the intrinsic microstructural self-organization of materials systems, and over the past few years there has been a burgeoning interest in the more general phase behaviour that cell components exhibit. In short, the materials of the cell may not be a 'blank slate', featureless until genes impose some order.

In particular, it is now widely recognized that proteins and nucleic acids may undergo phase separation to form compartments that act as organelles, even though they lack the membranes usually associated with organelles $^{1,2}$. These structures are often liquid droplets, although solid and gel phases are also found. They are crucial to cell function; dysregulation of the phase behaviour can lead to disease.

That picture is expanded by a new study by Mahamid et al. showing that lipids - which also form segregated droplet phases - have a rich phase behaviour in which transitions between liquid-crystalline states seem likely to have functional significance ${ }^{3}$. Lipid droplets have themselves been recognized for some time; they are surrounded by a phospholipid membrane, sequestering the lipids into what were long assumed to be passive storage compartments.

More recently, however, these droplets have been shown to play an active role in lipid metabolism ${ }^{4}$.

Mahamid et al. have taken a closer look at these lipid compartments using high-resolution cryo-electron microscopy. This technique arrests cells in a vitrified state, with their watery cytoplasm remaining amorphous, that resembles the native physiological state more closely than for cells fixed or dehydrated for conventional electron microscopy.

The researchers studied HeLa (human cancer) cells, in which rapid proliferation boosts the demand for free fatty-acid lipids, and in truth it remains to be seen how far the findings generalize to healthy cells.

They find that, under these conditions, lipid droplets have a complex structure in which an amorphous core is surrounded by a shell where the lipid molecules are arrayed in layers (up to 30 or so of them) with a spacing of $3.4-3.6 \mathrm{~nm}$ : a liquid-crystalline phase, composed of cholesteryl esters (CEs). This is a smectic phase: the molecules are orientationally ordered, but within each stacked layer they have no lateral positional order. This ordered state arises from the phase separation of CEs from triacylglycerols (TAGs), the other major component of the lipid droplets. That in turn depends on there being a sufficiently high

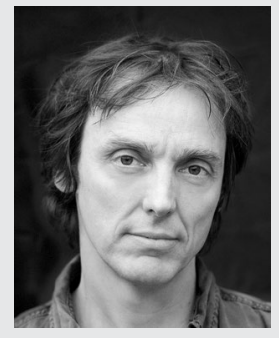

Philip Ball

CE concentration; in cells where TAG production is boosted or CE production lowered, the liquid-crystal phase isn't seen.

It is possible, then, that a transition between liquid-crystal and disordered phases in the droplets is not just a passive epiphenomenon of lipid concentration changes but also a functional response to shifts in the cell's lipid metabolism. The CE lipids will be more firmly sequestered in the ordered smectic phase, slowing exchange of these molecules with the surrounding environment and perhaps altering the rate at which they are metabolized and degraded. Could such changes play a role in disease states, such as atherosclerosis, where lipid droplets are known to be important? It's possible - and emphasizes the need to develop a more detailed picture of the materials science of the cell.

Published online: 21 August 2019

https://doi.org/10.1038/s41563-019-0474-5

References

1. Brangwynne, C. P. et al. Science 324, 1729-1732 (2009).

2. Boeynaems, S. et al. Trends Cell Biol. 28, 420-435 (2018).

3. Mahamid, J. et al. Proc. Natl Acad. Sci. USA. https://doi. org/10.1073/pnas.1903642116 (2019).

4. Fujimoto, T. \& Parton, R. G. Cold Spring Harb. Perspect. Biol. 3, a004838 (2011). 